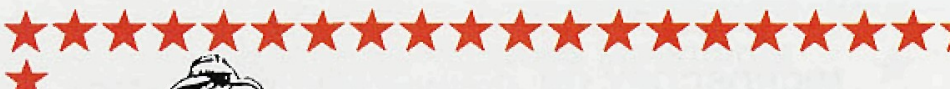 T) fume hood! Without good ventilation, you will start to notice rings around lights very quickly!

We mentioned this method in our paper:

Hawkins, H.K., M.L. Entman, J.Y. Zhu, K.A. Youker, K. Berens, M. Dore, and C.W. Smith. 1996. Acute inflammatory reaction after myocardial ischemic injury and reperfusion. Development and use of a neutrophilspecific antibody. Am. J. Pathol. 148(6):1957-1969.

Hal Hawkins, University of Texas Medical Branch

\section{A Hint for Fixing Tissues for Better Staining}

For beautiful colors in near perfectly preserved cells and connective tissue, immerse carefully cut pieces, no more than $2 \mathrm{~mm}$ in least dimension, ovemight in either Heidenhain's SUSA or Helly's fluid or Zenker's fluid without the acetic acid (which is the same as Helly's without the formalin). Wash thoroughly in water (tap water is OK) for several hours (overnight is sensible) and then dehydrate, clear, infiltrate, embed and cut paraffin sections

After dewaxing and hydrating the slides, put them in an iodine solution (Lugol's, Gram's or $0.5 \% \mathrm{I}_{2}$ in $95 \%$ ethanol), then in sodium thiosulfate $(2-6 \%)$ to remove the brown iodine color. Wash in running tap water for a minute to get rid of the chemicals. (This step is a must after any mercury-containing fix.)

Stain by any good, interesting method (meaning almost anything other than hematoxylin \& eosin).

Compare the result with that obtained with routine formaldehydefixed specimens. When comparing, think especially about what you believe to be the most important features seen with the microscope

John A. Kiernan, The University of Western Ontario

\section{A Procedure for LW Polymerization of Lowicryl or Similar Methacrylates}

1. Use polyethylene fiat embedding molds. These molds are made from the same type of material as BEEM capsules. The polyethylene molds are

transparent to the UV light, making the polymerization much more even.

2. Place the mold on a piece of cardboard that is wrapped in aluminum foil. The cardboard should be larger than the mold. This makes moving and transferring the mold much easier and less messy (see below).

3. Overfill all of the cavities in the mold to give a "positive meniscus" at each position. If some of the cavities don't have specimens in them, go ahead and fill the cavities anyway.

4. Cut a piece of Parafllm that is slightly larger than the mold. Then, beginning at one end of the mold, gently lay the Parafllm down on top of the mold. As you do this, the excess resin will run out of the mold cavities and get trapped under the Parafilm. Gradually lower the Parafilm down toward the other end of the mold, allowing the resin to fill the space between the mold and the Parafllm. This seals the complete top of the mold. The resin won't dissolve the Parafilm, but it will make it soft. Thermanox (plastic) cover slips can be used instead of Parafilm. Just lay the coverslips on top of the cavities, letting the excess resin mn run along the edges.

5. You can use tissues to absorb the excess resin as it runs down the outside of the mold. ( ${ }^{\star} \mathrm{CAUTION}:{ }^{\star \hbar}$ Be sure to wear gloves at this step, and at all steps when working with methacrylate resins - or any resin, for that matter!).

6. Transfer the filled molds to your polymerization apparatus. I make polymerization chambers out of cardboard boxes. Just make two cutouts: one on the top for the UV lamp, and one on the side for a door. Note! To make the polymerization even, cover all of the inner surfaces of the box with aluminum foil.

7. Let everything equilibrate at low temperature for $15-20$ minutes, turn on the UV lamp, and you're on your way to polymerized resin!

8. If you see "bubbles" or vortex-like "swirls" in the polymerized blocks, 